\title{
Data processing approaches in adopting single point-like emitter spectromicroscopy for mapping material characteristics of solid media
}

\author{
Alina Golovanova ${ }^{1,2, *}$ \\ ${ }^{1}$ Institute for Spectroscopy RAS, 108840 Troitsk, Moscow, Russia \\ ${ }^{2}$ Moscow State Pedagogical University, 119991 Moscow, Russia
}

\begin{abstract}
We report an algorithm for building topogram of dielectric properties of a thin film by data of spectromicroscopy of single emitters.
\end{abstract}

Spectromicroscopy of single quantum emitters has been recognized a powerful tool to study parameters of solid transparent mediums $[1,2]$. For example, in recent works it was proposed to use single fluorescent molecules as spectral probe by which it is possible to determine the effective local value of the refractive index $n$ [3].

Processing of experimental data obtained from the spectra and the fluorescence images of single molecules (SMs) in solid transparent matrices is known to have several problems which can be divided between physical-chemical and computational [4,5]. The first problem includes the presence of erroneous events, presents of other molecules, defects. The second problem includes huge or very small amount of data, hardware capabilities, etc. The problem considered in this work was a limited data and a significantly inhomogeneous spatial distribution of SMs over the plane of the sample. Note that these and related problems relates also to the spectromicroscopy of single semiconductor quantum dots [6]. The task of this study was to develop an algorithm for the correct transition from a discrete picture to a continuous distribution SMs data, to estimate the quality of such a transition and to construct a topogram based on SMs distributed somehow over the sample's plane.

The algorithm for building a topogram requires a certain data format. In our case, in the original data set, the local density of SMs in the sample plane fluctuates significantly, which makes direct averaging difficult. In order to eliminate this problem, the following algorithm was developed. The data plane is split by a lattice (grid) with a step $r$. Each node in the grid becomes the center of a circle with a fixed radius $R$. Each circle collects a number of SMs, while the minimum number of collected SMs depends on the selected $R$. The averaged data in each circle aria were used to give the average value of the effective refractive index of the film according to $[7,8]$. Each obtained value is attached to node with the coordinate XY. We have two key parameters: a grid step $r$ and a radius $R$. The radius must be quite big to include the minimum quantity of SMs and, at the same time, be limited so that averaging does not take place over all available SMs. If we choice too large $R$, we loss information about local changes in the structure. Consequently, with an optimal

* Corresponding author: golovanova@isan.troitsk.ru 
selection of these parameters, a more informative map of the values of $n$ can be constructed.

Such an analysis was carried out on the example the generated model array of effective refractive index values distributed randomly in the surface of the sample (with size 50 to 50 arbitrary units) (Fig.1.(a)). The values of $n$ are generated in accordance with the normal distribution law (the mathematical expectation is 1.5 , the standard deviation is 0.1 ). As a result of processing obtained array we is constructed a corresponding topogram (Fig.1.(b)).

For the transition from the discrete distribution of $n$ to the continuous one we used a standard interpolation packet based on radial basis functions (RBF) [9].

The algorithm was tested on the data obtained for SMs of an organic dye in a thin polycrystalline film.
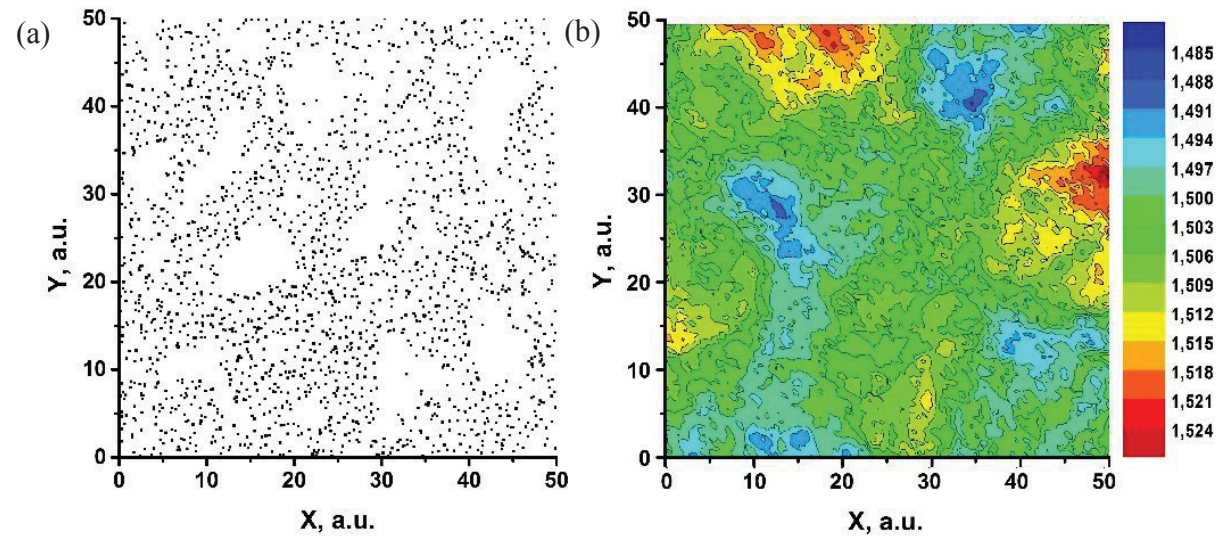

Fig. 1. (a) Model data distributed randomly in the surface of the sample with size 50 to 50 arbitrary units; (b) Topography of model data. Color scale shows the distribution of effective refractive index $n$ from 1.485 to 1.524 with step 0.003 .

Thus, the possibility of mapping the material characteristics (in this case n) of a continuous medium based on the discrete effective local $n$ values obtained with the use of point spectral probes (SMs) randomly distributed in the plane of a thin-film sample was shown. It is important to note that the developed algorithm can be used even if the local data is missing in some regions of the sample.

This work was supported by Grant of the Russian Science Foundation (\# 14-12-01415). Author is the member of the Leading Russian Scientific School (HШ-7035.2016.2).

\section{References}

[1] A.V. Naumov, Physics-Uspekhi 56, 605 (2013)

[2] A.V. Naumov, I.Y. Eremchev, A.A. Gorshelev, Eur. Phys. J. D 68, 348 (2014)

[3] T.A. Anikushina, M.G. Gladush, A.A. Gorsheleva, A.V. Naumov, Faraday Discuss. 184, 263 (2015)

[4] A.V. Naumov, A.A. Gorshelev, Y.G. Vainer, L. Kador, J. Kohler, Ang. Chemie. Int. ed. 51, 182 (2009)

[5] A.V. Naumov, A.A. Gorshelev, Y.G. Vainer, L. Kador, J. Kohler, Phys. Chem. Chem. Phys. 13, 1734 (2011)

[6] A.L. Shchukina, I.Y. Eremchev, A.V. Naumov, Phys. Rev. E 92, 032102 (2015)

[7] D.V. Kuznetsov, Vl.K. Roerich, M.G. Gladush, JETP 140(4), 742 (2011)

[8] M.G. Gladush, EPJ Web Conf. 132, 03007 (2017)

[9] M.D. Buhmann, Acta Numerica 9, 1 (2000) 